\title{
Acute Transverse Myelitis Associated with Severe Acute Respiratory Syndrome in Patient with COVID-19: A Case Report
}

\author{
Mohamad Khatib ${ }^{1}$, Ezeddin Alataresh ${ }^{1}$, Mohammad Alwraidat ${ }^{1}$, Amna Ahmed ${ }^{1}$, Ahmed \\ soliman Mohamed ${ }^{1}$, mohamed aboukamar ${ }^{1}$, Ahmad Abujaber ${ }^{1}$, and Abdulqadir Nashwan ${ }^{1}$ \\ ${ }^{1}$ Hamad Medical Corporation
}

September 16, 2020

\begin{abstract}
Acute transverse myelitis is a rare disease that can behave either in a benign course where it may end up in full neurologic recovery, or permanent neurologic sequelae and death. This report describes a 52-year-old male who presented with features of acute transverse myelitis associated with COVID-19.
\end{abstract}

Keywords: SARS-CoV-2, COVID-19, acute transverse myelitis, magnetic resonance imaging

Key Clinical Message

The authors urge clinicians to observe the signs and symptoms of acute transverse myelitis, and immediate initiation of a high dose of corticosteroids may help in ameliorating the symptoms, and hence the outcome.

\section{Background}

Coronavirus disease 2019 (COVID-19) is a disease caused by the severe acute respiratory syndrome coronavirus 2 (SARS-CoV-2) ${ }^{1}$, which was declared by The World Health Organization (WHO) as a pandemic on 11 March 2020. As of August 2020, more than 21 million cases have been reported globally; Qatar has recorded its first confirmed case of COVID-19 on February 29, 2020, and has a total of 114,809 confirmed cases, with a total of 192 deaths. ${ }^{2}$ Transverse myelitis is an inflammatory disorder with a heterogeneous pathology classified into acute or subacute spinal cord dysfunction and clinically featured by abnormal motor skills, sensory alterations, and disturbed bladder and bowel activities. ${ }^{3}$

This report describes an adult male who presented with features of acute transverse myelitis associated with COVID-19.

\section{Case Report}

We describe a 52 years old gentleman with a known case of diabetes mellitus (type II), presented to the emergency department of Hazm Mebaireek General Hospital in Doha, Qatar with symptoms suggestive of pneumonia associated with a lower abdominal pain and urine retention, Covid-19 was tested positive on the same day of admission.

KUB ultrasound showed right mild hydroureteronephrosis, left mild hydronephrosis, significant post-void residual urine $(873 \mathrm{cc})$, and mild enlargement of the prostate $(24 \mathrm{cc})$, hence urinary catheter was inserted, on the day of admission he reported lower limbs weakness, MRI spine and brain showed Subtle T2 hyperintense signals in the ventral horn of the grey matter in the thoracic cord T3-T10 (Figure 1), which represents viral myelitis with lower limb weakness. Lumbar puncture showed lymphocytic pleocytosis, normal protein, and glucose. COVID-19 PCR was negative in the Cerebrospinal fluid (CSF). Other viral panels, HIV 
serology, and autoimmune screening were negative. The baseline ECG was normal with sinus rhythm. Chest X-ray revealed right lower lobe infiltrate. Laboratory tests showed the following: Ferritin: 435.0 ug/L (Reference range $30 \mathrm{ug} / \mathrm{L}-553 \mathrm{ug} / \mathrm{L}$ ), Interleukin-6: $97 \mathrm{pg} / \mathrm{mL}$ (Reference range [?] $7 \mathrm{pg} / \mathrm{mL}$ ), CRP: 78.6 $\mathrm{mg} / \mathrm{L}$ (Reference range $0 \mathrm{mg} / \mathrm{L}-5 \mathrm{mg} / \mathrm{L}$ ), D-dimer: $23.57 \mathrm{mg} / \mathrm{L}$ (Reference range $0 \mathrm{mg} / \mathrm{L}-0.49 \mathrm{mg} / \mathrm{L}$ ). Therefore, intravenous steroid (Methylprednisolone $1 \mathrm{gm}$ daily for 3 days), acyclovir 700mg TID for 3 days, and a prophylactic dose of enoxaparin $40 \mathrm{mg}$ subcutaneous OD, were started. Limbs physiotherapy and occupational therapy were commenced.

On day 5 of hospital admission, the patient had a sudden cardiac arrest with an initial rhythm of pulseless electrical activity (PEA). Pulmonary embolism was highly suspected, ECG showed new incomplete right bundle branch block (RBBB), so reteplase rTPA $50 \mathrm{mg}$ IV bolus was given, he was admitted to the intensive care unit (ICU), where he had multiple cardiac arrests, which were not successfully resuscitated, eventually his death was declared on the $6^{\text {th }}$ day of hospital admission.

\section{Discussion}

Acute transverse myelitis (ATM) is characterized by localized inflammation of certain spinal cord segments. It is uncertain that it is due to direct viral invasion or by an autoimmune response to (SARS-CoV-2) virus. The diagnosis of ATM is made based on characteristic clinical findings in addition to serologic, MRI, and CSF analysis. The incidence rate of idiopathic ATM has been estimated to be between one and eight new cases per million annually. ${ }^{4}$

The incidence of neurologic involvement in patients diagnosed with SARS-CoV-2 is variable from 6 to $36.4 \%$. ${ }^{5}$ SARS-CoV-2 binds to ACE-2 receptors strongly in the lungs, heart, central nervous system, and skeletal muscles. ${ }^{6}$

The viral replication and activation of ACE-2 receptor in the CNS may trigger a systemic inflammatory response. ${ }^{6,7} \mathrm{IL}-6$, a proinflammatory cytokine, has been thought to mediate this response, and in our case, IL-6 was $(97 \mathrm{pg} / \mathrm{mL})$.

T2-weighted MRI is the imaging modality of choice to detect any spinal cord lesions; MRI's role is not limited to detect spinal cord lesions but also to rule out other differential diagnoses that may mimic the same clinical symptoms. Signal changes tend to affect the central region of the cord and involve more than two-thirds of the diameter of the cord as well as extend longitudinally over more than 1 segment in acute myelitis. The affected spinal cord segments appear hyperintense on T2 sequences with associated cord swelling. ${ }^{8,9}$

There is no curative treatment for transverse myelitis ${ }^{10}$; therefore, preventing or minimizing permanent neurological deficits is the main purpose of ATM's treatment. Treatment options include corticosteroid or plasmapheresis if there is an inadequate response to initial treatment.

The first case of acute myelitis due to SARS-CoV-2 was reported in Wuhan, China in March 2020. A male patient (66 years old) who had no known contact with patients with COVID-19 was presented with fever and fatigue for two days. Later, he was discharged to a rehabilitation facility to continue his treatment due to post-infection spinal cord involvement. ${ }^{11}$

The second case was reported in May 2020 in Denmark, for a young (28 years) female patient who presented with urinary retention, lower back pain, and generalized numbness. Intravenous corticosteroids and plasma exchange had significantly improved her symptoms. ${ }^{12}$

The third case was reported in June 2020, where a 61-year-old female patient presented with similar symptoms like our case; however, the ATM led to a permanent neurologic impairment requiring extensive rehabilitation. ${ }^{13}$

To our knowledge, this is the fourth reported case of ATM due to SARS-CoV-2 worldwide. Our case is a bit different where the patient devastatingly died during the index admission probably from SARS-CoV-2 related complications like acute pulmonary embolism, fulminant myocarditis, or cytokine storm. 


\section{Conclusion}

ATM has a wide range of presentations and it may lead to significant morbidity and mortality that require the clinicians to increase their index of suspicion to the diagnosis and its potentially life-threatening consequences. Immediate initiation of pulse steroids may help in ameliorating the neurologic symptoms. The absence of the SARS-CoV-2 RNA in the CSF does not exclude the diagnosis of ATM, and hence it postulates that immune-mediated post-inflammatory process took place in this context. While we learn more about the SARS-CoV-2, we must accept that there are many causes of death more than acute respiratory failure.

\section{Abbreviations}

ATM: acute transverse myelitis,

MRI: magnetic resonance image

COVID-19: coronavirus disease 2019

PEA: pulseless electrical activity

RBBB: right bundle branch block

\section{Declarations}

\section{Ethics approval and consent to participate}

The article describes a case report. Therefore, no additional permission from our Ethics Committee was required.

\section{Consent for publication}

The consent for publication was obtained.

\section{Availability of data and material}

All data generated or analyzed during this study are included in this published article.

\section{Competing interests}

The authors declare that they have no competing interests.

\section{Funding}

This study was not funded.

\section{Authors' contributions}

MYK, EMA, MAA, AAA, ASM, MRA, AAJ, AJN: Data Collection, Literature Search, Manuscript Preparation

All authors read and approved the final manuscript

\section{Acknowledgments}

We thank the patient for allowing us to share his case. Open Access funding provided by the Qatar National Library

\section{References}

1. Lai C-C, Shih T-P, Ko W-C, Tang H-J, Hsueh P-R. Severe acute respiratory syndrome coronavirus 2 (SARS-CoV-2) and corona virus disease-2019 (COVID-19): the epidemic and the challenges.International journal of antimicrobial agents. 2020:105924.

2. WHO. Coronavirus disease ( COVID-19): situation report, 209. 2020. 
3. Pandey S, Garg RK, Malhotra HS, et al. Etiologic spectrum and prognosis in noncompressive acute transverse myelopathies: An experience of 80 patients at a tertiary care facility. Neurology India.2018;66(1):65.

4. Durrani M, Kucharski K, Smith Z, Fien S. Acute Transverse Myelitis Secondary to Severe Acute Respiratory Syndrome Coronavirus 2 (SARS-CoV-2): A Case Report. Clinical Practice and Cases in Emergency Medicine. 2020;4(3):344.

5. Herman C, Mayer K, Sarwal A. Scoping review of prevalence of neurologic comorbidities in patients hospitalized for COVID-19.Neurology. 2020.

6. Mao L, Jin H, Wang M, et al. Neurologic manifestations of hospitalized patients with coronavirus disease 2019 in Wuhan, China.JAMA neurology. 2020;77(6):683-690.

7. $\mathrm{Wu} \mathrm{Y}, \mathrm{Xu} \mathrm{X}$, Chen Z, et al. Nervous system involvement after infection with COVID-19 and other coronaviruses. Brain, behavior, and immunity. 2020.

8. Lim PA. Transverse myelitis. Essentials of Physical Medicine and Rehabilitation. 2020:952.

9. Scotti G, Gerevini S. Diagnosis and differential diagnosis of acute transverse myelopathy. The role of neuroradiological investigations and review of the literature. Neurological Sciences.2001;22(2):S69-S73.

10. Lee DM, Jeon HS, Yoo WH. Transverse myelitis in a patient with primary antiphospholipid syndrome. Yonsei medical journal.2003;44(2):323-327.

11. Zhao K, Huang J, Dai D, Feng Y, Liu L, Nie S. Acute myelitis after SARS-CoV-2 infection: a case report. MedRxiv. 2020.

12. Sarma D, Bilello LA. A Case Report of Acute Transverse Myelitis Following Novel Coronavirus Infection. Clinical Practice and Cases in Emergency Medicine. 2020.

13. Valiuddin H, Skwirsk B, Paz-Arabo P. Acute Transverse Myelitis Associated with SARS-CoV-2: A Case-Report. Brain, Behavior, \&3 Immunity-Health. 2020.

Figure 1: MRI Spine showing T2 Hyperintense signals in the ventral horn of the grey matter in the thoracic cord T3-T10 


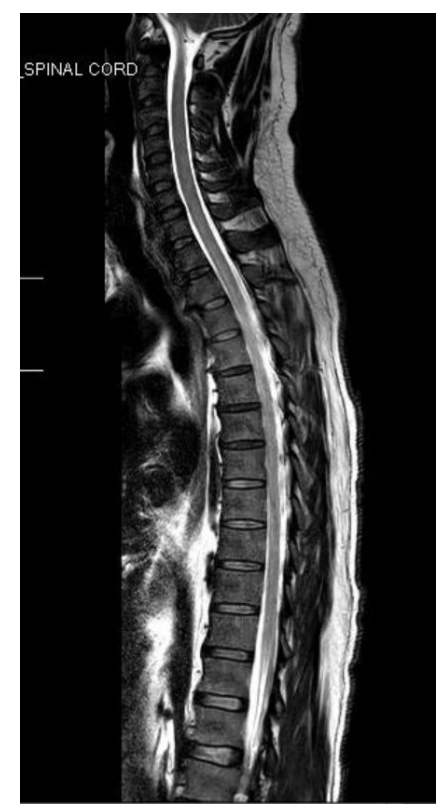

Figure 1: MRI Spine showing T2 Hyperintense signals in the ventral horn of the grey matter in the thoracic cord T3-T10 